TRANSACTIONS OF THE

AMERICAN MATHEMATICAL SOCIETY

Volume 363, Number 11, November 2011, Pages 5745-5759

S 0002-9947(2011)05204-9

Article electronically published on June 15, 2011

\title{
MEAN CURVATURE FLOW OF THE GRAPHS OF MAPS BETWEEN COMPACT MANIFOLDS
}

\author{
KUO-WEI LEE AND YNG-ING LEE
}

\begin{abstract}
We make several improvements on the results of M.-T. Wang (2002) and his joint paper with M.-P. Tsui (2004) concerning the long time existence and convergence for solutions of mean curvature flow in higher codimension. Both the curvature condition and lower bound of $* \Omega$ are weakened. New applications are also obtained.
\end{abstract}

\section{INTRODUCTION}

From the first variation formula of area for a submanifold in a Riemannian manifold, we can consider the mean curvature vector as the negative gradient of the area functional. The area of the submanifold will decrease most rapidly if we deform the submanifold in the direction of its mean curvature vector. Such a deformation is called mean curvature flow. It is a very natural way to find minimal submanifolds or canonical representatives. The study of mean curvature flow/curve shortening flow is very active and has had much advancement in the past thirty years. It started from the work of Brakke [1, and the paper of Huisken [3] opened a new era on the mean curvature flow of hypersurface. New developments were obtained in recent years on mean curvature flow in higher co-dimension. Since our work mainly focuses on generalizing the results in [7] and [8], we do not intend to list all important developments and papers on mean curvature flow here. Please refer to the papers [7, 8] and the references therein.

In this paper, we prove the following theorems:

Theorem 1. Let $\left(N_{1}, g\right)$ and $\left(N_{2}, h\right)$ be two compact Riemannian manifolds, and let $f$ be a smooth map from $N_{1}$ to $N_{2}$. Assume that $K_{N_{1}} \geq k_{1}$ and $K_{N_{2}} \leq k_{2}$ for two constants $k_{1}$ and $k_{2}$, where $K_{N_{1}}$ and $K_{N_{2}}$ are the sectional curvature of $N_{1}$ and $N_{2}$, respectively. Suppose either $k_{1} \geq 0, k_{2} \leq 0$, or $k_{1} \geq k_{2}>0$. Then the following results hold:

(i) If $\frac{\operatorname{det}\left(\left(g+f^{*} h\right)_{i j}\right)}{\operatorname{det}\left(g_{i j}\right)}<4$, then the mean curvature flow of the graph of $f$ remains the graph of a map and exists for all time.

(ii) Furthermore, if $k_{1}>0$, then the mean curvature flow converges smoothly to the graph of a constant map.

Received by the editors October 17, 2008 and, in revised form, September 11, 2009. 2010 Mathematics Subject Classification. Primary 53C44.

(C)2011 American Mathematical Society Reverts to public domain 28 years from publication 5745 
Theorem 2. Assume the same conditions as in Theorem 1. Then the following results hold:

(i) If $f$ is a smooth area-decreasing map from $N_{1}$ to $N_{2}$, then the mean curvature flow of the graph of $f$ remains the graph of an area-decreasing map and exists for all time.

(ii) Furthermore, if $k_{1}>0$, then the mean curvature flow converges smoothly to the graph of a constant map.

In Theorem 1 and Theorem 2 , we generalize the curvature conditions on $N_{1}$ and $N_{2}$ of the main theorems in [8] and [7] from constant sectional curvature to varied ones. Moreover, in Theorem 10 the upper bound on $\frac{\operatorname{det}\left(\left(g+f^{*} h\right)_{i j}\right)}{\operatorname{det}\left(g_{i j}\right)}$ in $[8$ ] is relaxed from 2 to 4 , which should also be observed from [7. However, since it is not mentioned and proved there, for completeness we treat this generalization as well. We also want to remark that the correct condition (which is related to $* \Omega$ ) in [8] should be $\frac{\sqrt{\operatorname{det}\left(g_{i j}\right)}}{\sqrt{\operatorname{det}\left(\left(g+f^{*} h\right)_{i j}\right)}}$ instead of $\frac{1}{\sqrt{\operatorname{det}\left(\left(g+f^{*} h\right)_{i j}\right)}}$.

We can apply Theorem 2 to show

Corollary 1. Let $N_{1}, N_{2}$ be compact manifolds and dim $N_{1} \geq 2$. Suppose that there exist Riemannian metrics $g_{1}$ and $g_{2}$ on $N_{1}$ and $N_{2}$ with sectional curvature $K_{N_{1}\left(g_{1}\right)}>0$ and $K_{N_{2}\left(g_{2}\right)} \leq 0$. Then any map from $N_{1}$ to $N_{2}$ must be homotopic to a constant map.

Corollary 2. Let $\left(N_{1}, g_{1}\right),\left(N_{2}, g_{2}\right)$ be compact Riemannian manifolds with $K_{N_{1}\left(g_{1}\right)}$ $\geq k_{1}, K_{N_{2}\left(g_{2}\right)} \leq k_{2}$, and both $k_{1}$ and $k_{2}$ are positive constants. If the 2-dilation of $f:\left(N_{1}, g_{1}\right) \rightarrow\left(N_{2}, g_{2}\right)$ is less than $\frac{k_{1}}{k_{2}}$, then $f$ is homotopic to a constant map.

We made most of the observations in this paper a few years ago and explained the arguments to M.-T. Wang and M.-P. Tsui in 2004 when the second author visited them at Columbia University. We thank M.-T. Wang for suggesting we write this paper and telling us of the interest in $k$-dilation maps. The authors also express their gratitude to the referee for a few helpful suggestions. A version of Theorem 2 in the pseudo-Riemannian case was obtained recently in [5].

To prove Theorems 1 and 2, we first need to show that the solution of mean curvature flow remains the graph of a map satisfying the same constraint as the initial map. This step depends on the curvature condition. Once we obtain the inequality in the first step, a similar argument as in 8 shows that the solution exists for all time. A refined inequality is needed to show that $* \Omega$ will converge to 1 as $t$ tends to infinity. We also need the curvature condition in this part. The last step, which is to show that the limit is a graph of a constant map, is the same as in 8 .

We list basic definitions and properties in $\S 2$ as preliminaries. Theorem 1 is proved in $\S 3$, and for completeness we also sketch the argument for the part which is similar to [8]. In $\S 4$, we discuss the area-decreasing case and prove Theorem 2 . The applications are given in $\S 5$.

\section{Preliminaries}

Assume that $N_{1}$ and $N_{2}$ are two compact Riemannian manifolds with metric $g$ and $h$, and of dimension $n$ and $m$, respectively. Let $f: N_{1} \rightarrow N_{2}$ be a smooth map 
and denote the graph by $\Sigma$. Then $\Sigma$ is an embedded submanifold in the product manifold $M=N_{1} \times N_{2}$ with $F=$ id. $\times f: N_{1} \rightarrow M$.

A smooth family $F_{t}: N_{1} \rightarrow M$ is called a mean curvature flow of $\Sigma$ if it satisfies

$$
\left\{\begin{array}{l}
\left(\frac{\partial F_{t}(x)}{\partial t}\right)^{\perp}=H(x, t), \\
F_{0}\left(N_{1}\right)=\Sigma,
\end{array}\right.
$$

where $H$ is the mean curvature vector of $F_{t}\left(N_{1}\right)=\Sigma_{t}$ and $(\cdot)^{\perp}$ denotes the projection onto the normal bundle $N \Sigma_{t}$ of $\Sigma_{t}$. By standard theories, the flow has short time existence.

Let $\Omega$ be a parallel $n$-form on $M$. We can evaluate this $n$-form on $\Sigma_{t}$. Choose orthonormal frames $\left\{e_{i}\right\}_{i=1}^{n}$ on $T \Sigma_{t}$ and $\left\{e_{\alpha}\right\}_{\alpha=n+1}^{n+m}$ on $N \Sigma_{t}$. The following evolution equation for $\Omega$ is derived by M.-T. Wang:

Proposition 1 ([8]). If $F_{t}$ is an n-dimensional mean curvature flow of $\Sigma$ in $M$ and $\Omega$ is a parallel $n$-form on $M$, then $\Omega_{1 \cdots n}=\Omega\left(e_{1}, \ldots, e_{n}\right)$ satisfies

$$
\begin{aligned}
\frac{\partial}{\partial t} \Omega_{1 \cdots n}= & \Delta \Omega_{1 \cdots n}+\Omega_{1 \cdots n} \sum_{\alpha, i, k}\left(h_{i k}^{\alpha}\right)^{2} \\
& -2 \sum_{\alpha<\beta, k}\left(\Omega_{\alpha \beta 3 \cdots n} h_{1 k}^{\alpha} h_{2 k}^{\beta}+\Omega_{\alpha 2 \beta \cdots n} h_{1 k}^{\alpha} h_{3 k}^{\beta}\right. \\
& \left.+\cdots+\Omega_{1 \cdots(n-2) \alpha \beta} h_{(n-1) k}^{\alpha} h_{n k}^{\beta}\right) \\
& -\sum_{\alpha, k}\left(\Omega_{\alpha 2 \cdots n} R_{\alpha k k 1}+\cdots+\Omega_{1 \cdots(n-1) \alpha} R_{\alpha k k n}\right),
\end{aligned}
$$

where $\Delta$ denotes the time-dependent Laplacian on $\Sigma_{t}, h_{i j}^{\alpha}=\left\langle\nabla_{e_{i}}^{M} e_{j}, e_{\alpha}\right\rangle$ is the second fundamental form, and $R$ is the curvature tensor of $M=N_{1} \times N_{2}$ with the product metric $g+h$.

Remark 1. Here we use the same convention as in [8] that

$$
\begin{aligned}
& R(X, Y) Z=-\nabla_{X} \nabla_{Y} Z+\nabla_{Y} \nabla_{X} Z+\nabla_{[X, Y]} Z, \\
& R_{i j k l}=\left\langle R\left(e_{k}, e_{l}\right) e_{i}, e_{j}\right\rangle
\end{aligned}
$$

and the sectional curvature is $K\left(e_{k}, e_{i}\right)=\left\langle R\left(e_{k}, e_{i}\right) e_{k}, e_{i}\right\rangle$, where $\left\{e_{i}\right\}$ are orthonormal.

Since $M=N_{1} \times N_{2}$ is a product manifold, the volume form $\Omega_{1}$ of $N_{1}$ can be extended as a parallel $n$-form on $M$. At any point $p$ on $\Sigma_{t}$, we have $* \Omega=$ $\Omega_{1}\left(e_{1}, \ldots, e_{n}\right)=\Omega_{1}\left(\pi_{1}\left(e_{1}\right), \ldots, \pi_{1}\left(e_{n}\right)\right)$, which is the Jacobian of the projection from $T_{p} \Sigma_{t}$ to $T_{\pi_{1}(p)} N_{1}$. By the implicit function theorem, we know $* \Omega>0$ near $p$ if and only if $\Sigma_{t}$ is locally a graph over $N_{1}$ near $p$.

When $\Sigma_{t}$ is the graph of $f_{t}: N_{1} \rightarrow N_{2}$, by the singular value decomposition theorem, there exist an orthonormal basis $\left\{a_{i}\right\}_{i=1}^{n}$ on $T_{\pi_{1}(p)} N_{1}$ and $\left\{a_{\alpha}\right\}_{\alpha=n+1}^{n+m}$ on $T_{\pi_{2}(p)} N_{2}$ so that $d f_{t}\left(a_{i}\right)=\lambda_{i} a_{n+i}$ for $1 \leq i \leq r$ and $d f_{t}\left(a_{i}\right)=0$ for $r \leq i \leq n$. Note that $r \leq \min (n, m)$ is the rank of $d f_{t}$ at $p$ and the $\lambda_{i}$ 's are the eigenvalues of $\sqrt{\left(d f_{t}\right)^{T} d f_{t}}$. Hence $\lambda_{i} \geq 0$ for all $i=1, \ldots, n$. We can use $\left\{a_{i}\right\}_{i=1}^{n}$ and $\left\{a_{\alpha}\right\}_{\alpha=n+1}^{n+m}$ to construct special orthonormal bases $\left\{E_{i}\right\}_{i=1}^{n}$ on $T_{p} \Sigma_{t}$ and $\left\{E_{\alpha}\right\}_{\alpha=n+1}^{n+m}$ on $N_{p} \Sigma_{t}$ 
as follows:

$$
\begin{aligned}
E_{i} & = \begin{cases}\frac{1}{\sqrt{1+\lambda_{i}^{2}}}\left(a_{i}+\lambda_{i} a_{n+i}\right) & \text { if } 1 \leq i \leq r, \\
a_{i} & \text { if } r+1 \leq i \leq n,\end{cases} \\
E_{n+q} & = \begin{cases}\frac{1}{\sqrt{1+\lambda_{q}^{2}}}\left(a_{n+q}-\lambda_{q} a_{q}\right) & \text { if } 1 \leq q \leq r, \\
a_{n+q} & \text { if } r+1 \leq q \leq m .\end{cases}
\end{aligned}
$$

Thus,

$$
* \Omega=\Omega_{1}\left(\pi_{1}\left(E_{1}\right), \ldots, \pi_{1}\left(E_{n}\right)\right)=\frac{1}{\sqrt{\prod_{i=1}^{n}\left(1+\lambda_{i}^{2}\right)}} .
$$

With these new bases (2) and (3), we can rewrite (11) as follows. This evolution equation is derived in $[8$, and here we express the formula in a general form.

Proposition 2 ([8]). Suppose $M=N_{1} \times N_{2}$ with the product metric $g+h$ and $\Omega$ is the parallel extension of the volume form of $N_{1}$. Let $\Sigma$ be an embedded submanifold in $M$ and a graph over $N_{1}$. If the mean curvature flow of $\Sigma$ is a graph over $N_{1}$, then $* \Omega$ satisfies the following equation:

$$
\begin{aligned}
\frac{\partial}{\partial t} * \Omega= & \Delta \Omega+* \Omega|A|^{2}+* \Omega\left(2 \sum_{k, i<j} \lambda_{i} \lambda_{j} h_{i k}^{n+j} h_{j k}^{n+i}-2 \sum_{k, i<j} \lambda_{i} \lambda_{j} h_{i k}^{n+i} h_{j k}^{n+j}\right) \\
& +* \Omega \sum_{i, k} \frac{\lambda_{i}^{2}}{\left(1+\lambda_{i}^{2}\right)\left(1+\lambda_{k}^{2}\right)}\left\langle R_{1}\left(a_{k}, a_{i}\right) a_{k}, a_{i}\right\rangle \\
& -* \Omega \sum_{i, k} \frac{\lambda_{i}^{2} \lambda_{k}^{2}}{\left(1+\lambda_{i}^{2}\right)\left(1+\lambda_{k}^{2}\right)}\left\langle R_{2}\left(a_{n+k}, a_{n+i}\right) a_{n+k}, a_{n+i}\right\rangle
\end{aligned}
$$

where $|A|^{2}$ denotes the norm square of the second fundamental form and where $R_{1}, R_{2}$ denote the curvature tensors on $N_{1}, N_{2}$ with metric $g, h$, respectively.

Proof. From the evolution equation (11) and bases (2), (3), one has

$$
\begin{aligned}
& \Omega_{1 \cdots \alpha \cdots \beta \cdots n} h_{i k}^{\alpha} h_{j k}^{\beta}=* \Omega \lambda_{i} \lambda_{j}\left(h_{i k}^{n+i} h_{j k}^{n+j}-h_{j k}^{n+i} h_{i k}^{n+j}\right), \\
& \Omega_{1 \cdots \alpha \cdots n}=-* \Omega \lambda_{i}, \\
& R_{(n+i) k k i}=\frac{-\lambda_{i} \lambda_{k}^{2}}{\left(1+\lambda_{i}^{2}\right)\left(1+\lambda_{k}^{2}\right)}\left\langle R_{2}\left(a_{n+k}, a_{n+i}\right) a_{n+i}, a_{n+k}\right\rangle \\
& \quad+\frac{\lambda_{i}}{\left(1+\lambda_{i}^{2}\right)\left(1+\lambda_{k}^{2}\right)}\left\langle R_{1}\left(a_{k}, a_{i}\right) a_{i}, a_{k}\right\rangle .
\end{aligned}
$$

The evolution equation (4) thus follows directly.

When $* \Omega>0$, one can consider the evolution equation of $\ln * \Omega$ instead and have the following. 
Proposition 3 ([7]). The evolution equation (4) can be rewritten as the form

$$
\begin{aligned}
\frac{\partial}{\partial t} \ln * \Omega= & \Delta \ln * \Omega+|A|^{2}+\sum_{i, k} \lambda_{i}^{2}\left(h_{i k}^{n+i}\right)^{2}+2 \sum_{k, i<j} \lambda_{i} \lambda_{j} h_{i k}^{n+j} h_{j k}^{n+i} \\
& +\sum_{i, k} \frac{\lambda_{i}^{2}}{\left(1+\lambda_{i}^{2}\right)\left(1+\lambda_{k}^{2}\right)}\left\langle R_{1}\left(a_{k}, a_{i}\right) a_{k}, a_{i}\right\rangle \\
& -\sum_{i, k} \frac{\lambda_{i}^{2} \lambda_{k}^{2}}{\left(1+\lambda_{i}^{2}\right)\left(1+\lambda_{k}^{2}\right)}\left\langle R_{2}\left(a_{n+k}, a_{n+i}\right) a_{n+k}, a_{n+i}\right\rangle .
\end{aligned}
$$

Proof. Since $\frac{\partial}{\partial t} \ln * \Omega=\frac{1}{* \Omega}\left(\frac{\partial}{\partial t} * \Omega\right)$, it implies $\frac{\partial}{\partial t} * \Omega=* \Omega\left(\frac{\partial}{\partial t} \ln * \Omega\right)$. Similarly, one has

$$
\Delta \ln * \Omega=\frac{\Delta * \Omega}{* \Omega}-\frac{|\nabla * \Omega|^{2}}{|* \Omega|^{2}}=\frac{\Delta * \Omega}{* \Omega}-\frac{\left|\Omega_{1 \cdots n, k}\right|^{2}}{|* \Omega|^{2}}=\frac{\Delta * \Omega}{* \Omega}-\left|\sum_{i, k} \lambda_{i} h_{i k}^{n+i}\right|^{2}
$$

or

$$
\Delta * \Omega=* \Omega(\Delta \ln * \Omega)+* \Omega\left(\sum_{i, k} \lambda_{i} h_{i k}^{n+i}\right)^{2} .
$$

Plugging these expressions into equation (44) and dividing $* \Omega$ on both sides, equation (51) is then obtained.

\section{Proof of Theorem 1}

Now we are ready to prove

Theorem 1. Let $\left(N_{1}, g\right)$ and $\left(N_{2}, h\right)$ be two compact Riemannian manifolds, and let $f$ be a smooth map from $N_{1}$ to $N_{2}$. Assume that $K_{N_{1}} \geq k_{1}$ and $K_{N_{2}} \leq k_{2}$ for two constants $k_{1}$ and $k_{2}$, where $K_{N_{1}}$ and $K_{N_{2}}$ are the sectional curvature of $N_{1}$ and $N_{2}$, respectively. Suppose either $k_{1} \geq 0, k_{2} \leq 0$, or $k_{1} \geq k_{2}>0$. Then the following results hold:

(i) If $\frac{\operatorname{det}\left(\left(g+f^{*} h\right)_{i j}\right)}{\operatorname{det}\left(g_{i j}\right)}<4$, then the mean curvature flow of the graph of $f$ remains the graph of a map and exists for all time.

(ii) Furthermore, if $k_{1}>0$, then the mean curvature flow converges smoothly to the graph of a constant map.

Proof of (i). For convenience, we write equation (5) as

$$
\frac{\partial}{\partial t} \ln * \Omega=\Delta \ln * \Omega+\mathrm{I}+\mathrm{II}
$$


where

$$
\begin{aligned}
\mathrm{I}= & \text { second fundamental form terms } \\
= & |A|^{2}+\sum_{i, k} \lambda_{i}^{2}\left(h_{i k}^{n+i}\right)^{2}+2 \sum_{k, i<j} \lambda_{i} \lambda_{j} h_{i k}^{n+j} h_{j k}^{n+i}, \\
\mathrm{II}= & \text { curvature tensor terms } \\
= & \sum_{i, k} \frac{\lambda_{i}^{2}}{\left(1+\lambda_{i}^{2}\right)\left(1+\lambda_{k}^{2}\right)}\left\langle R_{1}\left(a_{k}, a_{i}\right) a_{k}, a_{i}\right\rangle \\
& -\sum_{i, k} \frac{\lambda_{i}^{2} \lambda_{k}^{2}}{\left(1+\lambda_{i}^{2}\right)\left(1+\lambda_{k}^{2}\right)}\left\langle R_{2}\left(a_{n+k}, a_{n+i}\right) a_{n+k}, a_{n+i}\right\rangle \\
= & \sum_{i, k \neq i}\left(\frac{\lambda_{i}^{2}}{\left(1+\lambda_{i}^{2}\right)\left(1+\lambda_{k}^{2}\right)} K_{N_{1}}\left(a_{k}, a_{i}\right)-\frac{\lambda_{i}^{2} \lambda_{k}^{2}}{\left(1+\lambda_{i}^{2}\right)\left(1+\lambda_{k}^{2}\right)} K_{N_{2}}\left(a_{n+k}, a_{n+i}\right)\right) .
\end{aligned}
$$

If we can show there exists $\delta>0$ such that

$$
\frac{\partial}{\partial t} \ln * \Omega \geq \Delta \ln * \Omega+\delta|A|^{2},
$$

by the maximum principle (the minimum version), $\min _{\Sigma_{t}} \ln * \Omega$ is nondecreasing in $t$ and $* \Omega \geq \min _{\Sigma_{t=0}} * \Omega>0$. Thus $\Sigma_{t}$ remains the graph of a map $f_{t}: N_{1} \rightarrow N_{2}$ whenever the flow exists. Moreover, since

$$
* \Omega=\frac{\sqrt{\operatorname{det}\left(g_{i j}\right)}}{\sqrt{\operatorname{det}\left(\left(g+f^{*} h\right)_{i j}\right)}}=\frac{1}{\sqrt{\prod_{i=1}^{n}\left(1+\lambda_{i}^{2}\right)}},
$$

we have $\min _{\Sigma_{t=0}} * \Omega>\frac{1}{2}$, and thus $\min _{\Sigma_{t}} * \Omega>\frac{1}{2}$ along the flow as well.

So we first aim at proving equation (77). From (8) and the compactness of $N_{1}$, it follows that $\prod_{i=1}^{n}\left(1+\lambda_{i}^{2}\right) \leq 4-\varepsilon$ on $\Sigma_{t=0}$ for some $\varepsilon>0$. By continuity and the short time existence of the flow, the solution remains the graph of a map and satisfies $\prod_{i=1}^{n}\left(1+\lambda_{i}^{2}\right) \leq 4-\frac{\varepsilon}{2}$ for small $t$.

In particular, when $i \neq j,\left(1+\lambda_{i}^{2}\right)\left(1+\lambda_{j}^{2}\right) \leq 4-\frac{\varepsilon}{2}$. By mean inequality, we have $\left|\lambda_{i} \lambda_{j}\right| \leq 1-\delta$ for $\delta=\frac{\varepsilon}{8}>0, i \neq j$. Thus

$$
\begin{aligned}
\mathrm{I} & \geq \delta|A|^{2}+(1-\delta) \sum_{i, j, k}\left(h_{j k}^{n+i}\right)^{2}-2(1-\delta) \sum_{k, i<j}\left|h_{j k}^{n+i} h_{i k}^{n+j}\right| \\
& \geq \delta|A|^{2}+(1-\delta) \sum_{k, i<j}\left(\left|h_{j k}^{n+i}\right|-\left|h_{i k}^{n+j}\right|\right)^{2} \\
& \geq \delta|A|^{2} .
\end{aligned}
$$

For curvature tensor terms:

(a) If $k_{1} \geq 0, k_{2} \leq 0$, we have

$$
\mathrm{II} \geq \sum_{i, k \neq i}\left(\frac{\lambda_{i}^{2}}{\left(1+\lambda_{i}^{2}\right)\left(1+\lambda_{k}^{2}\right)} k_{1}-\frac{\lambda_{i}^{2} \lambda_{k}^{2}}{\left(1+\lambda_{i}^{2}\right)\left(1+\lambda_{k}^{2}\right)} k_{2}\right) \geq 0 .
$$


(b) If $k_{1} \geq k_{2}>0$, then

$$
\begin{aligned}
\mathrm{II} & \geq \sum_{i, k \neq i}\left(\frac{\lambda_{i}^{2}}{\left(1+\lambda_{i}^{2}\right)\left(1+\lambda_{k}^{2}\right)} k_{1}-\frac{\lambda_{i}^{2} \lambda_{k}^{2}}{\left(1+\lambda_{i}^{2}\right)\left(1+\lambda_{k}^{2}\right)} k_{2}\right) \\
& \geq \sum_{i, k \neq i}\left(\frac{\lambda_{i}^{2}-\lambda_{i}^{2} \lambda_{k}^{2}}{\left(1+\lambda_{i}^{2}\right)\left(1+\lambda_{k}^{2}\right)}\right) k_{2}=\sum_{i<k}\left(\frac{\lambda_{i}^{2}+\lambda_{k}^{2}-2 \lambda_{i}^{2} \lambda_{k}^{2}}{\left(1+\lambda_{i}^{2}\right)\left(1+\lambda_{k}^{2}\right)}\right) k_{2} .
\end{aligned}
$$

Since $\left|\lambda_{i} \lambda_{k}\right|<1$,

$$
\begin{aligned}
\lambda_{i}^{2}+\lambda_{k}^{2}-2 \lambda_{i}^{2} \lambda_{k}^{2} & =\left(\lambda_{i}-\lambda_{k}\right)^{2}+2 \lambda_{i} \lambda_{k}-2 \lambda_{i}^{2} \lambda_{k}^{2} \\
& =\left(\lambda_{i}-\lambda_{k}\right)^{2}+2 \lambda_{i} \lambda_{k}\left(1-\lambda_{i} \lambda_{k}\right) \geq 0 .
\end{aligned}
$$

Hence II $\geq 0$.

Therefore (7) holds for small $t$. It follows that in fact $* \Omega \geq \min _{\Sigma_{t=0}} * \Omega>\frac{1}{\sqrt{4-\varepsilon}}$ for small $t$. Thus we can continue the same argument to conclude that the solution remains the graph of a map and satisfies $* \Omega \geq \min _{\Sigma_{t=0}} * \Omega>\frac{1}{\sqrt{4-\varepsilon}}$ whenever the flow exists.

Then by choosing $u=\frac{\ln * \Omega-\ln \Omega_{0}+c}{-\ln \Omega_{0}+c}$ with $c>0$ to replace $* \Omega$, the same proof as in 8 ] leads to the long-time existence of the flow. The only thing needed in the proof is equation (77).

The idea goes as follows: To detect a possible singularity, say $\left(y_{0}, t_{0}\right)$, one first isometrically embeds $M$ into $\mathbb{R}^{N}$ by Nash's theorem, and introduces the backward heat kernel from Huisken [4]

$$
\rho_{y_{0}, t_{0}}=\frac{1}{\left(4 \pi\left(t_{0}-t\right)\right)^{\frac{n}{2}}} \mathrm{e}^{-\frac{\left|y-y_{0}\right|^{2}}{4\left(t_{0}-t\right)}} .
$$

Direct computation and using equation (7) gives

$$
\frac{d}{d t} \int_{\Sigma_{t}}(1-u) \rho_{y_{0}, t_{0}} d \mu_{t} \leq C-\delta \int_{\Sigma_{t}}|A|^{2} \rho_{y_{0}, t_{0}} d \mu_{t}
$$

for some $C>0$. Therefore, $\lim _{t \rightarrow t_{0}} \int_{\Sigma_{t}}(1-u) \rho_{y_{0}, t_{0}} d \mu_{t}$ exists. Consider the parabolic dilation $D_{\lambda}$ at $\left(y_{0}, t_{0}\right)$, that is,

$$
(y, t) \stackrel{D_{\lambda}}{\longmapsto}\left(\lambda\left(y-y_{0}\right), \lambda^{2}\left(t-t_{0}\right)\right),
$$

and set $s=\lambda^{2}\left(t-t_{0}\right)$. Denote the corresponding submanifold and volume form after dilation by $\Sigma_{s}^{\lambda}$ and $d \mu_{s}^{\lambda}$, respectively. Because $u$ is invariant under parabolic dilation, inequality (10) becomes

$$
\frac{d}{d s} \int_{\Sigma_{s}^{\lambda}}(1-u) \rho_{0,0} d \mu_{s}^{\lambda} \leq \frac{C}{\lambda^{2}}-\delta \int_{\Sigma_{s}^{\lambda}} \rho_{0,0}|A|^{2} d \mu_{s}^{\lambda} .
$$

With further discussion from (11), one can find $\lambda_{j} \rightarrow \infty$ and $s_{j} \rightarrow-1$ such that

$$
\int_{\Sigma_{s_{j}}^{\lambda_{j}} \cap K}|A|^{2} d \mu_{s_{j}}^{\lambda_{j}} \rightarrow 0 \text { as } j \rightarrow \infty
$$

for any compact set $K$. One can conclude that $\Sigma_{s_{j}}^{\lambda_{j}} \rightarrow \Sigma_{-1}^{\infty}$ as a Radon measure and $\Sigma_{-1}^{\infty}$ is the graph of a linear function with further investigation. Therefore,

$$
\lim _{t \rightarrow t_{0}} \int \rho_{y_{0}, t_{0}} d \mu_{t}=\lim _{j \rightarrow \infty} \int \rho_{0,0} d \mu_{s_{j}}^{\lambda_{j}}=1 .
$$


This implies that $\left(y_{0}, t_{0}\right)$ is a regular point by White's theorem in 9 , which is a contradiction. Thus no singularity can occur along the flow. We refer to 8 for the detailed argument.

Proof of (ii). We use the same expression as in (6) and will first show that there exists $c_{0}>0$ which depends on $\varepsilon, k_{1}, n$ such that

$$
\mathrm{II} \geq c_{0} \sum_{i=1}^{n} \lambda_{i}^{2} \geq c_{0} \ln \left(\prod_{i=1}^{n}\left(1+\lambda_{i}^{2}\right)\right)=-2 c_{0} \ln * \Omega .
$$

(a) If $k_{1}>0$, and $k_{2} \leq 0$, we have

$$
\begin{aligned}
\mathrm{II} & \geq \sum_{i, k \neq i}\left(\frac{\lambda_{i}^{2}}{\left(1+\lambda_{i}^{2}\right)\left(1+\lambda_{k}^{2}\right)} k_{1}-\frac{\lambda_{i}^{2} \lambda_{k}^{2}}{\left(1+\lambda_{i}^{2}\right)\left(1+\lambda_{k}^{2}\right)} k_{2}\right) \\
& \geq \sum_{i, k \neq i} \frac{\lambda_{i}^{2} k_{1}}{\left(1+\lambda_{i}^{2}\right)\left(1+\lambda_{k}^{2}\right)} \\
& \geq \frac{k_{1}(n-1)}{4} \sum_{i=1}^{n} \lambda_{i}^{2} \\
& \geq \frac{k_{1}(n-1)}{4} \sum_{i=1}^{n} \ln \left(1+\lambda_{i}^{2}\right),
\end{aligned}
$$

since $\frac{1}{\left(1+\lambda_{i}^{2}\right)\left(1+\lambda_{k}^{2}\right)} \geq \frac{1}{\prod_{i=1}^{n}\left(1+\lambda_{i}^{2}\right)} \geq \frac{1}{4}$ and $\lambda_{i}^{2} \geq \ln \left(1+\lambda_{i}^{2}\right)$. Hence we can take $c_{0}=\frac{k_{1}(n-1)}{4}$.

(b) If $k_{1} \geq k_{2}>0$, we need to estimate curvature terms more carefully. Recall

$$
\begin{aligned}
\mathrm{II} & \geq \sum_{i, k \neq i}\left(\frac{\lambda_{i}^{2}}{\left(1+\lambda_{i}^{2}\right)\left(1+\lambda_{k}^{2}\right)} k_{1}-\frac{\lambda_{i}^{2} \lambda_{k}^{2}}{\left(1+\lambda_{i}^{2}\right)\left(1+\lambda_{k}^{2}\right)} k_{2}\right) \\
& \geq \sum_{i, k \neq i}\left(\frac{\lambda_{i}^{2}-\lambda_{i}^{2} \lambda_{k}^{2}}{\left(1+\lambda_{i}^{2}\right)\left(1+\lambda_{k}^{2}\right)}\right) k_{1}=\sum_{i<k}\left(\frac{\lambda_{i}^{2}+\lambda_{k}^{2}-2 \lambda_{i}^{2} \lambda_{k}^{2}}{\left(1+\lambda_{i}^{2}\right)\left(1+\lambda_{k}^{2}\right)}\right) k_{1} .
\end{aligned}
$$

As observed in the proof of (i), we have $\left|\lambda_{i} \lambda_{k}\right|<1-\frac{\varepsilon}{4}$ for all $t \geq 0$. Thus,

$$
\lambda_{i}^{2}+\lambda_{k}^{2}-2 \lambda_{i}^{2} \lambda_{k}^{2}=\lambda_{i} \lambda_{k}\left(\lambda_{i}-\lambda_{k}\right)^{2}+\left(1-\lambda_{i} \lambda_{k}\right)\left(\lambda_{i}^{2}+\lambda_{k}^{2}\right) \geq \frac{\varepsilon}{4}\left(\lambda_{i}^{2}+\lambda_{k}^{2}\right) .
$$

Therefore,

$$
\mathrm{II} \geq \frac{\varepsilon k_{1}}{16} \sum_{i<k}\left(\lambda_{i}^{2}+\lambda_{k}^{2}\right)=\frac{\varepsilon k_{1}(n-1)}{16} \sum_{i=1}^{n} \lambda_{i}^{2} \geq \frac{\varepsilon k_{1}(n-1)}{16} \sum_{i=1}^{n} \ln \left(1+\lambda_{i}^{2}\right) .
$$

We can take $c_{0}=\frac{\varepsilon k_{1}(n-1)}{16}$.

Hence we can rewrite (6) as

$$
\frac{\partial}{\partial t} \ln * \Omega \geq \Delta \ln * \Omega-2 c_{0} \ln * \Omega .
$$


Consider a function $f(t)$ which depends only on $t$ and satisfies

$$
\left\{\begin{array}{l}
\frac{d}{d t} f(t)=-2 c_{0} f(t), \\
f(0)=\min _{\Sigma_{t=0}} \ln * \Omega,
\end{array}\right.
$$

which gives $f(t)=f(0) \mathrm{e}^{-2 c_{0} t}$. From the inequality (14) and (15), we have

$$
\frac{\partial}{\partial t}(\ln * \Omega-f(t)) \geq \Delta(\ln * \Omega-f(t))-2 c_{0}(\ln * \Omega-f(t)) .
$$

Because $\min _{\Sigma_{t=0}}(\ln * \Omega-f(t)) \geq 0$, by the maximum principle, we have

$$
\min _{\Sigma_{t>0}}(\ln * \Omega-f(t)) \geq 0 .
$$

Hence $0 \geq \ln * \Omega \geq f(0) \mathrm{e}^{-2 c_{0} t}$ on $\Sigma_{t \geq 0}$. Letting $t \rightarrow \infty$, it gives $* \Omega \rightarrow 1$. Then one can apply the same argument as in 8 to conclude that the solution converges smoothly to a constant map at infinity. We outline the proof of this fact in the next paragraph.

Given $\varepsilon_{1}>0$, there exists $T$ such that $* \Omega>\frac{1}{\sqrt{1+\varepsilon_{1}}}$ for $t>T$. It implies $\sum_{i} \lambda_{i}^{2}<\varepsilon_{1}$ for $t>T$. The same method as in (9) and taking $\delta$ larger, for example $\delta=\frac{1}{2}$, gives

$$
\frac{\partial}{\partial t} * \Omega \geq \Delta * \Omega+\frac{1}{2} * \Omega|A|^{2}
$$

The evolution inequality for the second fundamental form is

$$
\frac{\partial}{\partial t}|A|^{2} \leq \Delta|A|^{2}-2|\nabla A|^{2}+K_{1}|A|^{4}+K_{2}|A|^{2}+K_{3}
$$

for some constants $K_{1}, K_{2}, K_{3}$ that depend on the curvature tensor of $M$ and its covariant derivatives. The $K_{1}|A|^{4}$ term will cause some trouble, but one can consider the evolution inequality of $(* \Omega)^{-2 p}|A|^{2}$, which is

$$
\begin{aligned}
& \frac{\partial}{\partial t}\left((* \Omega)^{-2 p}|A|^{2}\right) \\
\leq & \Delta\left((* \Omega)^{-2 p}|A|^{2}\right)+2(* \Omega)^{-2 p} \nabla\left((* \Omega)^{-2 p}\right) \cdot \nabla\left((* \Omega)^{-2 p}|A|^{2}\right) \\
& +(* \Omega)^{-2 p}\left(|A|^{4}\left(C_{1}-p+2 p(2 p-1) n \varepsilon_{1}\right)+C_{2}\right) .
\end{aligned}
$$

Choose $\varepsilon_{1}$ small, and a suitable $p=p\left(n, \varepsilon_{1}\right)$ so that the coefficient of $(* \Omega)^{-2 p}|A|^{4}$ is negative. By the maximum principle, one gets an upper bound of $|A|^{2}$. Integrating (16) and (17), we obtain $\int_{\Sigma_{t}}|A|^{2} d \mu_{t} \rightarrow 0$ and also $\int_{\Sigma_{t}}|A|^{4} d \mu_{t} \rightarrow 0$ as $t \rightarrow \infty$ by the boundedness of $|A|^{2}$. Since $|A|^{2}$ is uniformly bounded, evolution inequality (17) implies $\frac{\partial}{\partial t}|A|^{2} \leq \Delta|A|^{2}+C_{3}$ for some constant $C_{3}$. For any positive function $u(x, t)$ satisfying $u_{t} \leq \Delta u+f(x, t)$ in a parabolic region $Q_{R}=B_{R}\left(x_{0}, t_{0}\right) \times\left(t_{0}-R^{2}, t_{0}+R^{2}\right)$, one has the following estimate [10, Theorem 4.4.2]

$$
\sup _{Q_{\frac{R}{2}}} u \leq c_{1} R^{-\frac{n}{2}-1}\|u\|_{L^{2}\left(Q_{R}\right)}+c_{2} R^{2}\|f\|_{L^{\infty}\left(Q_{R}\right)} .
$$


For any $\varepsilon>0$ small enough, one can find $T_{1}$ such that $\int_{\Sigma_{t}}|A|^{4} d \mu_{t} \leq \varepsilon^{n+2}$ for $t>T_{1}$. Take $R=\varepsilon$, then (18) implies

$$
|A|^{2}(x, t) \leq \sqrt{2} c_{1} \varepsilon+c_{2} \varepsilon^{2} C_{3} \quad \text { for all } t>T_{1}+\varepsilon^{2} \text { and } x \in \Sigma_{t} .
$$

Hence $\max _{\Sigma_{t}}|A|^{2} \rightarrow 0$ as $t \rightarrow \infty$. It implies that the mean curvature flow of $\Sigma$ converges to a totally geodesic submanifold of $M$. Since $* \Omega \rightarrow 1$ as $t \rightarrow \infty$, we have $\left|d f_{t}\right| \rightarrow 0$ and the limit is a constant map.

Remark 2. When $n=1$, we have $k_{1}=0$ and (ii) cannot apply. In fact, term II vanishes in this case, and one cannot obtain the convergence using the same method.

\section{The AREA-DECREASING CASE}

In this section, we mainly follow the discussion and setup in [7]. Consider a parallel symmetric two tensor $S$ on $M$ defined as

$$
S(X, Y)=g\left(\pi_{1}(X), \pi_{1}(Y)\right)-h\left(\pi_{2}(X), \pi_{2}(Y)\right)
$$

where $\pi_{1}$ and $\pi_{2}$ are the projections into $T N_{1}$ and $T N_{2}$, respectively. The same calculation as for $* \Omega$ leads to the following evolution equation for $S$ on $\Sigma_{t}$, which appears in [7,

$$
\begin{aligned}
\left(\frac{\partial}{\partial t}-\Delta\right) S_{i j}= & -h_{i l}^{\alpha} h_{k k}^{\alpha} S_{l j}-h_{j l}^{\alpha} h_{k k}^{\alpha} S_{l i}+R_{k i k \alpha} S_{\alpha j}+R_{k j k \alpha} S_{\alpha i} \\
& +h_{k l}^{\alpha} h_{k i}^{\alpha} S_{l j}+h_{k l}^{\alpha} h_{k j}^{\alpha} S_{l i}-2 h_{k i}^{\alpha} h_{k j}^{\beta} S_{\alpha \beta}
\end{aligned}
$$

where $S_{i j}=S\left(e_{i}, e_{j}\right), S_{\alpha i}=S\left(e_{\alpha}, e_{i}\right), S_{\alpha \beta}=S\left(e_{\alpha}, e_{\beta}\right), i, j=1, \ldots, n ; \alpha, \beta=n+$ $1, \ldots, n+m$.

One can simplify the above equation in terms of evolving orthonormal frames. Suppose that $\left\{e_{i}\right\}_{i=1}^{n}$ is a basis for the tangent bundle $T \Sigma_{t}$ and $\left\{e_{\alpha}\right\}_{\alpha=n+1}^{n+m}$ is a basis for the normal bundle $N \Sigma_{t}$. Denote

$$
\begin{aligned}
\bar{g}_{i j} & =g\left(\pi_{1}\left(e_{i}\right), \pi_{1}\left(e_{j}\right)\right)+h\left(\pi_{2}\left(e_{i}\right), \pi_{2}\left(e_{j}\right)\right), \\
\bar{g}_{\alpha \beta} & =g\left(\pi_{1}\left(e_{\alpha}\right), \pi_{1}\left(e_{\beta}\right)\right)+h\left(\pi_{2}\left(e_{\alpha}\right), \pi_{2}\left(e_{\beta}\right)\right),
\end{aligned}
$$

and $\bar{g}^{i j}$ the inverse metric of $\bar{g}_{i j}$. Now assume that $\bar{F}=\left\{F_{1}, \ldots, F_{a}, \ldots, F_{n}\right\}$ are orthonormal frames on $T_{p} \Sigma_{t}$. We evolve $\bar{F}$ by the formula

$$
\frac{\partial}{\partial t} F_{a}^{i}=\bar{g}^{i j} \bar{g}_{\alpha \beta} h_{k j}^{\alpha} H^{\beta} F_{a}^{k}
$$

where $\alpha$ and $\beta$ are in the normal direction and $H^{\beta}$ is the $\beta$ component of the mean curvature vector. 
Let $S_{a b}=S_{i j} F_{a}^{i} F_{b}^{j}=S\left(F_{a}, F_{b}\right)$ be the component of $S$ in $\bar{F}$. Then $S_{a b}$ satisfies the following equation:

$$
\left(\frac{\partial}{\partial t}-\Delta\right) S_{a b}=R_{c a c \alpha} S_{\alpha b}+R_{c b c \alpha} S_{\alpha a}+h_{c d}^{\alpha} h_{c a}^{\alpha} S_{d b}+h_{c d}^{\alpha} h_{c b}^{\alpha} S_{d a}-2 h_{c a}^{\alpha} h_{c b}^{\beta} S_{\alpha \beta} .
$$

We remark that when we use the bases (2) and (3), the expression of $S$ is

$$
S=S\left(E_{i}, E_{j}\right)_{1 \leq i, j \leq n+m}=\left(\begin{array}{cccc}
B & 0 & D & 0 \\
0 & I_{(n-r) \times(n-r)} & 0 & 0 \\
D & 0 & -B & 0 \\
0 & 0 & 0 & -I_{(m-r) \times(m-r)}
\end{array}\right),
$$

where $B$ and $D$ are $r$ by $r$ matrices with

$$
B_{i j}=S\left(E_{i}, E_{j}\right)=\frac{1-\lambda_{i}^{2}}{1+\lambda_{i}^{2}} \delta_{i j} \quad \text { and } \quad D_{i j}=S\left(E_{i}, E_{n+j}\right)=-\frac{2 \lambda_{i}}{1+\lambda_{i}^{2}} \delta_{i j} .
$$

A map $f: N_{1} \rightarrow N_{2}$ is called area-decreasing if

$$
\left|\bigwedge^{2} d f\right|(x)=\sup _{|u \wedge v|=1}\left|\left(\bigwedge^{2} d f\right)(u \wedge v)\right|=\sup _{|u \wedge v|=1}|d f(u) \wedge d f(v)|<1 .
$$

In the bases (2) and (3), the area-decreasing condition is equivalent to

$$
\left|\bigwedge^{2} d f\right|(x)=\sup _{i<j} \lambda_{i} \lambda_{j}<1 \Leftrightarrow\left|\lambda_{i} \lambda_{j}\right|<1 \forall i \neq j .
$$

On the other hand, the sum of any two eigenvalues of $S$ is

$$
\frac{1-\lambda_{i}^{2}}{1+\lambda_{i}^{2}}+\frac{1-\lambda_{j}^{2}}{1+\lambda_{j}^{2}}=\frac{2\left(1-\lambda_{i}^{2} \lambda_{j}^{2}\right)}{\left(1+\lambda_{i}^{2}\right)\left(1+\lambda_{j}^{2}\right)} .
$$

Thus, the area-decreasing condition is equivalent to the two positivity of $S$.

Since $S$ is bilinear, by the Riesz representation theorem, we can identify $S$ with a self-adjoint operator (still denoted by $S$ ). Hence, for the orthonormal frame $\bar{F}$, we have $S_{a b}=S\left(F_{a}, F_{b}\right)=\bar{g}\left(S\left(F_{a}\right), F_{b}\right)$, which implies $S\left(F_{a}\right)=S_{a b} F_{b}$.

With this identification, we can construct a new self-adjoint operator $S^{[2]}=S \otimes$ $1+1 \otimes S$ on $T_{p} \Sigma_{t} \wedge T_{p} \Sigma_{t}$, which is defined by $S^{[2]}\left(w_{1} \wedge w_{2}\right)=S\left(w_{1}\right) \wedge w_{2}+w_{1} \wedge S\left(w_{2}\right)$. If $\mu_{1} \leq \cdots \leq \mu_{n}$ are the eigenvalues of $S$ with the corresponding eigenvectors $v_{1}, \ldots, v_{n}$, then $S^{[2]}$ has eigenvalues $u_{i_{1}}+u_{i_{2}}$ with eigenvectors $v_{i_{1}} \wedge v_{i_{2}}, i_{1} \leq i_{2}$. Thus, the positivity of $S^{[2]}$ is equivalent to the area-decreasing condition. Similarly, for the metric $\bar{g}$, we can construct a self-adjoint operator $\bar{g}^{[2]}=\bar{g} \otimes 1+1 \otimes \bar{g}$.

Note that $\left\{F_{a} \wedge F_{b}\right\}_{a<b}$ form an orthonormal basis for $\bigwedge^{2} T \Sigma_{t}$ and

$$
\begin{aligned}
S^{[2]}\left(F_{a} \wedge F_{b}\right) & =S\left(F_{a}\right) \wedge F_{b}+F_{a} \wedge S\left(F_{b}\right)=S_{a c} F_{c} \wedge F_{b}+F_{a} \wedge S_{a c} F_{c} \\
& =\sum_{c<d}\left(S_{a c} \delta_{b d}+S_{b d} \delta_{a c}-S_{a d} \delta_{b c}-S_{b c} \delta_{a d}\right) F_{c} \wedge F_{d}, \\
\bar{g}^{[2]}\left(F_{a} \wedge F_{b}\right) & =\sum_{c<d}\left(2 \delta_{a c} \delta_{b d}-2 \delta_{a d} \delta_{b c}\right) F_{c} \wedge F_{d} .
\end{aligned}
$$


We can improve the main theorem in $[7$ to the following

Theorem 2. Let $\left(N_{1}, g\right)$ and $\left(N_{2}, h\right)$ be two compact Riemannian manifolds, and let $f$ be a smooth map from $N_{1}$ to $N_{2}$. Assume that $K_{N_{1}} \geq k_{1}$ and $K_{N_{2}} \leq k_{2}$ for two constants $k_{1}$ and $k_{2}$, where $K_{N_{1}}$ and $K_{N_{2}}$ are the sectional curvature of $N_{1}$ and $N_{2}$, respectively. Suppose either $k_{1} \geq 0, k_{2} \leq 0$, or $k_{1} \geq k_{2}>0$. Then the following results hold:

(i) If $f$ is a smooth area-decreasing map from $N_{1}$ to $N_{2}$, then the mean curvature flow of the graph of $f$ remains the graph of an area-decreasing map and exists for all time.

(ii) Furthermore, if $k_{1}>0$, then the mean curvature flow converges smoothly to the graph of a constant map.

Proof of (i). Notice that we already proved in $\$ 3$ that $\Sigma_{t}$ remains the graph of a map under the assumption whenever the flow exists. Now we want to prove that the area-decreasing property is also preserved along the mean curvature flow. Since the initial map is area-decreasing, there exists $\varepsilon>0$ such that $S^{[2]}-\varepsilon \bar{g}^{[2]} \geq 0$. We want to show that the property $S^{[2]}-\varepsilon \bar{g}^{[2]}$ is preserved along the mean curvature flow. Let $M_{\eta}=S^{[2]}-\varepsilon \bar{g}^{[2]}+\eta t \bar{g}^{[2]}$. Suppose the mean curvature flow exists on $[0, T)$. Consider any $T_{1}<T$; it suffices to show that $M_{\eta}>0$ on $\left[0, T_{1}\right]$ for all $\eta<\frac{\varepsilon}{2 T_{1}}$. If it does not hold, there will be a first time that $0<t_{0}<T_{1}, M_{\eta}$ is nonnegative definite, and there is a null eigenvector $V=V^{a b} F_{a} \wedge F_{b}$ for $M_{\eta}$ at some point $x_{0} \in \Sigma_{t_{0}}$. We extend $V$ to a parallel vector field in a neighborhood of $x_{0}$ along geodesics emanating out of $x_{0}$, and define $V$ on $[0, T)$ independent of $t$.

Define a function $f=M_{\eta}(V, V)$. Then the function $f$ has the following properties at $\left(x_{0}, t_{0}\right)$ :

(F1) $f=0$

( $V$ is the null-eigenvector).

(F2) $\nabla f=0$

(At $t=t_{0}, f$ attains minimum on $x_{0}$ ).

(F3) $\left(\frac{\partial}{\partial t}-\Delta\right) f \leq 0 \quad\left(\right.$ At $t=t_{0}, f$ attains minimum on $\left.x_{0}\right)$.

At $\left(x_{0}, t_{0}\right)$, we choose the orthonormal basis $\left\{F_{a}\right\}$ as $\left\{E_{i}\right\}$ in (2) and rearrange them such that the singular values $\lambda_{i}$ satisfy $\lambda_{1} \geq \lambda_{2} \geq \cdots \geq \lambda_{n} \geq 0$. Thus,

$$
S_{n n}=\frac{1-\lambda_{n}^{2}}{1+\lambda_{n}^{2}} \geq \cdots \geq S_{22}=\frac{1-\lambda_{2}^{2}}{1+\lambda_{2}^{2}} \geq S_{11}=\frac{1-\lambda_{1}^{2}}{1+\lambda_{1}^{2}}
$$

Hence the null eigenvector must be $V=E_{1} \wedge E_{2}$. From (F1), it follows that $f=S_{11}+S_{22}+2\left(\eta t_{0}-\varepsilon\right)=0$ at $\left(x_{0}, t_{0}\right)$, which implies $S_{11}+S_{22}=2\left(\varepsilon-\eta t_{0}\right)>0$. Thus, we have

$$
\lambda_{1} \lambda_{2}<1, \quad \text { and } \quad \lambda_{i}<1 \quad \text { for } \quad i \geq 2 .
$$

Use (19) to evolve $\left\{F_{a}\right\}$. Then at $\left(x_{0}, t_{0}\right)$, direct computation gives

$$
\begin{aligned}
\left(\frac{\partial}{\partial t}-\Delta\right) f= & 2 \eta+2 R_{k 1 k \alpha} S_{\alpha 1}+2 R_{k 2 k \alpha} S_{\alpha 2} \\
& +2 h_{k j}^{\alpha} h_{k 1}^{\alpha} S_{j 1}+2 h_{k j}^{\alpha} h_{k 2}^{\alpha} S_{j 2}-2 h_{k 1}^{\alpha} h_{k 1}^{\beta} S_{\alpha \beta}-2 h_{k 2}^{\alpha} h_{k 2}^{\beta} S_{\alpha \beta} \\
= & 2 \eta+\mathrm{I}+\mathrm{II},
\end{aligned}
$$


where

$$
\begin{aligned}
& \mathrm{I}=\text { curvature tensor terms } \\
& =2 R_{k 1 k \alpha} S_{\alpha 1}+2 R_{k 2 k \alpha} S_{\alpha 2}=2 R_{k 1 k(n+1)} S_{(n+1) 1}+2 R_{k 2 k(n+2)} S_{(n+2) 2} \\
& =\sum_{k \neq 1} \frac{2 \lambda_{1}^{2}}{\left(1+\lambda_{k}^{2}\right)\left(1+\lambda_{1}^{2}\right)^{2}}\left\langle R_{1}\left(a_{k}, a_{1}\right) a_{k}, a_{1}\right\rangle \\
& +\sum_{k \neq 2} \frac{2 \lambda_{2}^{2}}{\left(1+\lambda_{k}^{2}\right)\left(1+\lambda_{2}^{2}\right)^{2}}\left\langle R_{1}\left(a_{k}, a_{2}\right) a_{k}, a_{2}\right\rangle \\
& -\sum_{k \neq 1} \frac{2 \lambda_{k}^{2} \lambda_{1}^{2}}{\left(1+\lambda_{k}^{2}\right)\left(1+\lambda_{1}^{2}\right)^{2}}\left\langle R_{2}\left(a_{k}, a_{1}\right) a_{k}, a_{1}\right\rangle \\
& -\sum_{k \neq 2} \frac{2 \lambda_{k}^{2} \lambda_{2}^{2}}{\left(1+\lambda_{k}^{2}\right)\left(1+\lambda_{2}^{2}\right)^{2}}\left\langle R_{2}\left(a_{k}, a_{2}\right) a_{k}, a_{2}\right\rangle \\
& \geq \sum_{k \neq 1} \frac{2 \lambda_{1}^{2}}{\left(1+\lambda_{k}^{2}\right)\left(1+\lambda_{1}^{2}\right)^{2}} k_{1}+\sum_{k \neq 2} \frac{2 \lambda_{2}^{2}}{\left(1+\lambda_{k}^{2}\right)\left(1+\lambda_{2}^{2}\right)^{2}} k_{1} \\
& -\sum_{k \neq 1} \frac{2 \lambda_{k}^{2} \lambda_{1}^{2}}{\left(1+\lambda_{k}^{2}\right)\left(1+\lambda_{1}^{2}\right)^{2}} k_{2}-\sum_{k \neq 2} \frac{2 \lambda_{k}^{2} \lambda_{2}^{2}}{\left(1+\lambda_{k}^{2}\right)\left(1+\lambda_{2}^{2}\right)^{2}} k_{2} . \\
& \text { II }=\text { second fundamental form terms } \\
& =2 h_{k j}^{\alpha} h_{k 1}^{\alpha} S_{j 1}+2 h_{k j}^{\alpha} h_{k 2}^{\alpha} S_{j 2}-2 h_{k 1}^{\alpha} h_{k 1}^{\beta} S_{\alpha \beta}-2 h_{k 2}^{\alpha} h_{k 2}^{\beta} S_{\alpha \beta} .
\end{aligned}
$$

For curvature tensor terms I:

(a) If $k_{1} \geq 0, k_{2} \leq 0$, we have $\mathrm{I} \geq 0$.

(b) $\underline{\text { If } k_{1} \geq k_{2}>0}$, then

$$
\begin{aligned}
& \mathrm{I} \geq k_{1}\left(\sum_{k \neq 1} \frac{2 \lambda_{1}^{2}-2 \lambda_{k}^{2} \lambda_{1}^{2}}{\left(1+\lambda_{k}^{2}\right)\left(1+\lambda_{1}^{2}\right)^{2}}+\sum_{k \neq 2} \frac{2 \lambda_{2}^{2}-2 \lambda_{k}^{2} \lambda_{2}^{2}}{\left(1+\lambda_{k}^{2}\right)\left(1+\lambda_{2}^{2}\right)^{2}}\right) \\
& =k_{1}\left(\frac{2 \lambda_{1}^{2}-2 \lambda_{2}^{2} \lambda_{1}^{2}}{\left(1+\lambda_{2}^{2}\right)\left(1+\lambda_{1}^{2}\right)^{2}}+\frac{2 \lambda_{2}^{2}-2 \lambda_{1}^{2} \lambda_{2}^{2}}{\left(1+\lambda_{1}^{2}\right)\left(1+\lambda_{2}^{2}\right)^{2}}\right. \\
& \left.+\sum_{k \geq 3} \frac{2 \lambda_{1}^{2}-2 \lambda_{k}^{2} \lambda_{1}^{2}}{\left(1+\lambda_{k}^{2}\right)\left(1+\lambda_{1}^{2}\right)^{2}}+\sum_{k \geq 3} \frac{2 \lambda_{2}^{2}-2 \lambda_{k}^{2} \lambda_{2}^{2}}{\left(1+\lambda_{k}^{2}\right)\left(1+\lambda_{2}^{2}\right)^{2}}\right) \\
& \geq k_{1}\left(\frac{2 \lambda_{1}^{2}+2 \lambda_{2}^{2}-4 \lambda_{2}^{2} \lambda_{1}^{2}}{\left(1+\lambda_{1}^{2}\right)^{3}}\right)+\sum_{k \geq 3} k_{1}\left(\frac{2 \lambda_{1}^{2}\left(1-\lambda_{k}^{2}\right)}{\left(1+\lambda_{k}^{2}\right)\left(1+\lambda_{1}^{2}\right)^{2}}+\frac{2 \lambda_{2}^{2}\left(1-\lambda_{k}^{2}\right)}{\left(1+\lambda_{k}^{2}\right)\left(1+\lambda_{2}^{2}\right)^{2}}\right) \\
& \left.\geq k_{1}\left(\frac{2\left(\lambda_{1}-\lambda_{2}\right)^{2}+4 \lambda_{1} \lambda_{2}\left(1-\lambda_{1} \lambda_{2}\right)}{\left(1+\lambda_{1}^{2}\right)^{3}}\right) \quad \text { (here we use (22) }\right) \\
& \geq 0 \text { (here we use (22).) }
\end{aligned}
$$

Since the second fundamental form terms do not involve curvatures, II is nonnegative as proved in [7]. Since both $\mathrm{I} \geq 0$ and II $\geq 0$ at $\left(x_{0}, t_{0}\right)$, we have $\left(\frac{\partial}{\partial t}-\Delta\right) f \geq 2 \eta>0$ at $\left(x_{0}, t_{0}\right)$, which contradicts (F3). Thus the area-decreasing property is preserved by the mean curvature flow. We can also apply the same 
proof to obtain long-time existence as in $\$ 3$ because the inequality (7) holds as long as there exists $\delta>0$ such that $\left|\lambda_{i} \lambda_{j}\right|<1-\delta$ for $i \neq j$.

Proof of (ii). From the proof of (i), we know there exists $\varepsilon>0$ such that $S^{[2]}-$ $\varepsilon \bar{g}^{[2]} \geq 0$ for all $t \geq 0$. This condition is equivalent to

$$
\frac{1-\lambda_{i}^{2} \lambda_{j}^{2}}{\left(1+\lambda_{i}^{2}\right)\left(1+\lambda_{j}^{2}\right)} \geq \varepsilon \quad \text { for all } i \neq j
$$

In particular, we have

$$
\begin{aligned}
& \varepsilon\left(1+\lambda_{i}^{2}\right) \leq \frac{1-\lambda_{i}^{2} \lambda_{j}^{2}}{1+\lambda_{j}^{2}} \leq 1 \Rightarrow 1+\lambda_{i}^{2} \leq \frac{1}{\varepsilon} \quad \text { for all } i, \text { and } \\
& 1-\lambda_{i}^{2} \lambda_{j}^{2} \geq \varepsilon \Rightarrow\left|\lambda_{i} \lambda_{j}\right| \leq \sqrt{1-\varepsilon} \leq 1-\frac{1}{2} \varepsilon .
\end{aligned}
$$

These are the only estimates needed in the proof of convergence in Theorem 1. The same argument shows that there exists $c_{0}=c_{0}\left(\varepsilon, k_{1}, n\right)>0$ such that

$$
\mathrm{II} \geq c_{0} \sum_{i=1}^{n} \lambda_{i}^{2} \geq c_{0} \ln \left(\prod_{i=1}^{n}\left(1+\lambda_{i}^{2}\right)\right)=-2 c_{0} \ln * \Omega .
$$

This gives us the inequality (14), and the rest of the proof is the same as in Theorem 1.

\section{Application}

Corollary 1. Let $N_{1}, N_{2}$ be compact manifolds and dim $N_{1} \geq 2$. Suppose that there exist Riemannian metrics $g_{1}$ and $g_{2}$ on $N_{1}$ and $N_{2}$ with sectional curvature $K_{N_{1}\left(g_{1}\right)}>0$ and $K_{N_{2}\left(g_{2}\right)} \leq 0$. Then any map from $N_{1}$ to $N_{2}$ must be homotopic to a constant map.

Proof. For any given map $f: N_{1} \rightarrow N_{2}$, we can consider the singular value decomposition of $d f$ with respect to $g_{1}$ and $g_{2}$. Denote the corresponding singular values by $\lambda_{1}, \ldots, \lambda_{n}$. Since $N_{1}$ is compact, there exists a positive constant $L$ such that $\lambda_{i} \lambda_{j} \leq L$. Define a new metric $\bar{g}_{1}=2 L g_{1}$ on $N_{1}$. The singular values of $d f$ with respect to $\bar{g}_{1}$ and $g_{2}$ will be $\bar{\lambda}_{1}=\frac{\lambda_{1}}{\sqrt{2 L}}, \ldots, \bar{\lambda}_{n}=\frac{\lambda_{n}}{\sqrt{2 L}}$. Therefore, we have $\bar{\lambda}_{i} \bar{\lambda}_{j} \leq \frac{1}{2}<1$ and $K_{N_{1}\left(\bar{g}_{1}\right)}>0$. Applying the mean curvature flow to the graph of $f$ in $\left(N_{1}, \bar{g}_{1}\right) \times\left(N_{2}, g_{2}\right)$, by Theorem 2 we conclude that $f$ is homotopic to a constant map.

For general cases, we can obtain the null homotopic property in terms of 2dilation. Recall that the 2-dilation (or more generally, $k$-dilation) of a map $f$ between $N_{1}$ and $N_{2}$ is said to be at most $D$ if $f$ maps each 2-dimensional ( $k$ dimensional) submanifold in $N_{1}$ with volume $V$ to an image with volume at most $D V$. The 2-dilation can also be defined in terms of $d f$, which is equal to the supremum of the norm $\left|\bigwedge^{2} d f\right|$.

We have the following corollary:

Corollary 2. Let $\left(N_{1}, g_{1}\right),\left(N_{2}, g_{2}\right)$ be compact Riemannian manifolds with $K_{N_{1}\left(g_{1}\right)}$ $\geq k_{1}, K_{N_{2}\left(g_{2}\right)} \leq k_{2}$, and both $k_{1}$ and $k_{2}$ are positive constants. If the 2 -dilation of $f:\left(N_{1}, g_{1}\right) \rightarrow\left(N_{2}, g_{2}\right)$ is less than $\frac{k_{1}}{k_{2}}$, then $f$ is homotopic to a constant map. 
Proof. Consider the metrics $\bar{g}_{1}=k_{1} g_{1}$ and $\bar{g}_{2}=k_{2} g_{2}$. Then the sectional curvatures satisfy $K_{N_{1}\left(\bar{g}_{1}\right)} \geq 1, K_{N_{2}\left(\bar{g}_{2}\right)} \leq 1$, and the map $f:\left(N_{1}, \bar{g}_{1}\right) \rightarrow\left(N_{2}, \bar{g}_{2}\right)$ satisfies $\left|\bigwedge^{2} d f\right|<\frac{k_{1}}{k_{2}} \cdot \frac{k_{2}}{k_{1}}=1$, which is an area-decreasing mapping. By Theorem 2, $f$ is homotopic to a constant map.

Assume $\left(N_{1}, g_{1}\right)$ has nonnegative Ricci curvature and $\operatorname{dim} N_{1}=2$. A classical result in harmonic map theory tells us that there exists $\varepsilon>0$ such that if a harmonic map $f:\left(N_{1}, g_{1}\right) \rightarrow\left(N_{2}, g_{2}\right)$ satisfies $E(f)=\int_{N_{1}}\|d f\|^{2}<\varepsilon$, then $f$ is a constant map. As a final application of Theorem 2, one can prove a similar result. The idea is first to obtain the pointwise bound of $d f$ by the total energy and then apply Corollary 2 to conclude that $f$ is homotopic to a constant map when $K\left(g_{1}\right)$ > 0 . Such a pointwise estimate is obtained by Schoen [6] when $\operatorname{dim} N_{1}=2, f$ is harmonic, and the energy is sufficiently small in small balls. We remark that this argument works in higher dimension whenever the pointwise estimate is obtained.

\section{REFERENCES}

[1] K.A. Brakke, The motion of a surface by its mean curvature. Mathematical Notes, Princeton University Press, 1978. MR485012 (82c:49035)

[2] L. Guth, Homotopically non-trivial maps with small $k$-dilation. arXiv:0709.1241v1

[3] G. Huisken, Flow by mean curvature of convex surfaces into spheres. J. Differential Geom. 20 (1984), no. 1, 237-266. MR772132 (86j:53097)

[4] G. Huisken, Asymptotic behavior for singularities of the mean curvature flow. J. Differential Geom. 31 (1990), no. 1, 285-299. MR:1030675 (90m:53016)

[5] Guanghan Li; Isabel M.C. Salavessa, Mean curvature flow of spacelike graphs in PseudoRiemannian manifolds. arXiv:0804.0783

[6] R. Schoen, Analytic aspects of the harmonic map problem, Seminar on Nonlinear Partial Differential Equations, ed. S. S. Chern, Springer-Verlag, 1985, 321-358. MR765241 (86b:58032)

[7] M.-P. Tsui; M.-T. Wang, Mean curvature flows and isotopy of maps between spheres. Comm. Pure Appl. Math. 57 (2004), no. 8, 1110-1126. MR2053760(2005b:53110)

[8] M.-T. Wang, Long-time existence and convergence of graphic mean curvature flow in arbitrary codimension. Invent. Math. 148 (2002), no. 3, 525-543. MR1908059 (2003b:53073)

[9] B. White, A local regularity theorem for classical mean curvature flow. Ann. of Math. (2) 161 (2005), no. 3, 1487-1519. MR2180405(2006i:53100)

[10] Z. Wu; J. Yin; C. Wang, Elliptic and parabolic equations. World Scientific Publishing Co. Pte. Ltd., Hackensack, NJ, 2006. MR2309679 (2007m:35003)

Institute of Mathematics, Academia Sinica, Taipei, Taiwan

E-mail address: d93221007@ntu.edu.tw

Department of Mathematics, National Taiwan University, Taipei, Taiwan - and National Center for Theoretical Sciences, Taipei Office, National Taiwan University, TAIPEI, TAIWAN

E-mail address: yilee@math.ntu.edu.tw 